\title{
Los agroecosistemas cafetaleros modernos y su relación con la conservación de mariposas en paisajes fragmentados
}

\author{
Oscar Pérez-García ${ }^{1 *}$, Tamara J. Benjamin² \& Diego Enrique Tobar L. ${ }^{3}$ \\ 1. Centro Interdisciplinario de Investigación para el Desarrollo Integral Regional (CIIDIR), Unidad Oaxaca, Instituto \\ Politécnico Nacional, 71230, Santa Cruz Xoxocotlán, Oaxaca, México; ospeg@yahoo.com.mx \\ 2. College of Agriculture, Purdue University, 47907-2010, West Lafayette, Indiana, USA; tamara17@purdue.edu \\ 3. Programa de Ganadería y Manejo del Medio Ambiente (GAMMA), Centro Agronómico Tropical de Investigación y \\ Enseñanza (CATIE), 7170, Turrialba, Costa Rica; dtobar@catie.ac.cr \\ * Correspondencia
}

\author{
Recibido 20-VII-2017. Corregido 26-X-2017. Aceptado 25-XI-2017.
}

\begin{abstract}
Modern coffee agroecosystems and their relationship with butterflies conservation in fragmented landscapes. Land use change by the intensification of agriculture contributes to tropical forest fragmentation and biodiversity loss. In this sense, in comparison with traditional agroforestry systems (AFS), modern AFS are considered irrelevant for the conservation of biodiversity of tropical forest fragments. However, there is an increasing trend toward the establishment of different types of modern AFS. Owing to the sensitivity to environmental changes, we evaluated the biodiversity of butterflies in AFS with Coffea arabica and Erythrina poeppigiana (CP), and intermixed with Musa spp. (CPM) or Cordia alliodora (the only native species of AFS) (CPL), as well as premontane very humid forest fragments in the Volcánica Central-Talamanca Biological Corridor, Costa Rica. In six representative sites of each system we set up three parallel $80 \mathrm{~m}$ line-transects, separated by a distance of $25 \mathrm{~m}$. Each coffee farm and forest fragment (FR) was surveyed during 1.5 hours to record butterflies. All sites were sampled once a month from May to July 2007. We obtained 4.5 hours of sampling/ site, and 27 hours of sampling/habitat. Both CP and CPM together had a butterflies' species composition which differed from CPL and FR. The indicator species of both CP and CPM together were Hermeuptychia hermes and Anartia jatrophae which are typical of open areas. CPL resulted with the species of secondary forests Heliconius erato petiverana and Pareuptychia ocirrhoe as indicators. In contrast, FR resulted with several indicator species typical of closed canopy forests such as Pierella helvina incanescens, Caligo eurilochus, and Morpho peleides limpida. Moreover, the richness of species was similar among coffee AFS. Furthermore, the richness of species of each coffee AFS was lower in comparison with FR. However, among coffee AFS, CPL harbored higher diversity $\left(\operatorname{Exp}\left(H^{\prime}\right)\right)$ of forest butterflies than CP. Also, CPM was higher in abundance of frugivorous butterflies in relation with CP and CPL. In fragmented forests, coffee farms with exotic shade species combined at least with a native tree or fruit species, can be a better habitat for forest butterflies than those dominated by an exotic monospecific shade. Rev. Biol. Trop. 66(1): 394-402. Epub 2018 March 01.
\end{abstract}

Key words: agroforestry systems; coffee matrix; guilds; habitat preferences; indicator species; Papilionoidea.

El cambio de uso del suelo por la intensificación de la agricultura, contribuye con la fragmentación de los bosques tropicales y la pérdida de la biodiversidad, sobre todo de ecorregiones prioritarias para la conservación (Myers, Mittermeier, Mittermeier, \& da Fonseca, 2000; Dobrovolski, Diniz-Filho, Dias Loyola, \& De Marco Junior, 2011). Sin embargo, estudios recientes indican que en paisajes fragmentados, una proporción considerable de la biodiversidad original puede persistir dentro de las áreas agrícolas, si éstas mantienen cierta cobertura arbórea a través de sistemas agroforestales (SAF) (Kremen, 2015). En este sentido, uno de los SAF de gran importancia económica mundial es la producción de café bajo sombra (Jha et al., 2014). Diversos estudios han evidenciado que los SAF con café 
ayudan en gran medida a la conservación de la biodiversidad que los monocultivos de café sin sombra (De Beenhouwer, Aerst, \& Honnay, 2013). En particular, entre los SAF con café, los sistemas tradicionales de policultivos multiestrato han significado mayor importancia para la conservación de la diversidad biológica que los sistemas modernos con sombra monoespecífica (Mas \& Dietsch, 2003; De Beenhouwer et al., 2013).

Actualmente, existe una tendencia creciente al establecimiento de SAF modernos con café, que no han sido evaluados en estudios comparativos de biodiversidad (Jha et al., 2014). Estos nuevos agroecosistemas cafetaleros, incluyen variedades de café de alto rendimiento (p. ej., Coffea arabica L. var. Caturra o Catuai) y árboles de sombra con especies exóticas. Por ejemplo, en Mesoamérica es común el uso de sombras monoespecíficas con las especies exóticas Erythrina poeppigiana (Walp.) O. F. Cook o Grevillea robusta A. Cunn. ex R. Br. (Somarriba et al., 2004). En este sentido, uno de los grupos taxonómicos altamente sensibles a los cambios de temperatura, humedad y radiación solar por la perturbación de los bosques tropicales es el de las mariposas diurnas (Lepidoptera: Rhopalocera) (Bonebrake, Ponisio, Boggs, \& Ehrlich, 2010). A pesar de ser buenos indicadores de perturbación de los bosques, las mariposas diurnas han sido poco estudiadas en SAF modernos con café.

Debido a la sensibilidad ante cambios en la cobertura arbórea, se evaluó la biodiversidad de mariposas diurnas en tres SAF modernos con café y en los fragmentos de bosque en el Corredor Biológico Volcánica Central-Talamanca (CBVCT) en Costa Rica. En particular, se determinó la composición de especies de mariposas diurnas en SAF basados en $C$. arabica y E. poeppigiana y en combinación con Musa spp. o Cordia alliodora (la única especie nativa de los SAF). Además, se evaluó la riqueza de mariposas en general y la abundancia, riqueza y diversidad por gremios alimenticios y preferencias de hábitat. Esto, con la finalidad de identificar qué SAF cafetaleros contribuyen con la conservación de mariposas de los bosques tropicales de Mesoamérica. De esta manera, se espera llenar el vacío de conocimiento acerca de la capacidad de nuevos SAF para la conservación biológica en paisajes fragmentados.

\section{MATERIALES Y MÉTODOS}

Descripción del área de estudio: El estudio se realizó en el CBVCT localizado en los cantones de Turrialba y Jiménez, Cartago,

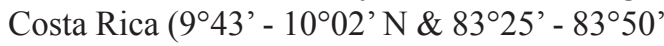
W). La zona presenta una precipitación media anual de $2650 \mathrm{~mm}$ y una temperatura media anual de $21.8^{\circ} \mathrm{C}$ (Canet, 2003). La topografía tiene pendientes que varían entre 30 y $60 \%$. En la zona predominan los siguientes usos del suelo: cobertura boscosa (40\% del CBVCT), pastizales $(24 \%)$, cafetales $(14 \%)$ y el resto por otros usos (Murrieta, 2006). La zonas de vida predominantes son el bosque muy húmedo premontano (53\% del CBVCT) y el bosque pluvial premontano (22\%) según la clasificación de Holdridge (Canet, 2003).

Selección de sitios: Se seleccionaron 18 cafetales correspondientes a seis parcelas de cada uno de los siguientes SAF: café-poró (CP: C. arabica-E. poeppigiana), café-porómusa (CPM: C. arabica-E. poeppigiana-Musa spp.) y café-poró-laurel (CPL: C. arabica-E. poeppigiana-C. alliodora). Adicionalmente, se seleccionaron seis fragmentos de bosque (FR) caracterizados por las especies arbóreas nativas Vismia macrophylla Kunth, Vochysia allenii Standl. \& L.O. Williams y Miconia punctata (Desr.) D. Don ex DC (Murrieta, 2006). Los SAF con café y los FR estuvieron distribuidos entre 600 a 1000 msnm en el bosque muy húmedo premontano en su totalidad. Solamente se seleccionaron parcelas de pequeños productores quienes manejan de 3000 a 5000 cafetos $\mathrm{ha}^{-1}$ (C. arabica var. Caturra), un descope y un desrame de sombra (E. poeppigiana) al año, trampeo de plagas, podas fitosanitarias del cafeto y aplicación de herbicidas.

Los tres SAF estuvieron integrados por la sombra arbórea exótica E. poeppigiana 
(Fabaceae) con una altura media de $5 \mathrm{~m}$ (Florian, 2005). El sistema CP normalmente mantiene una densidad media de 349 árboles ha $^{-1}$ (Florian, 2005). En CPM se combinan dos especies exóticas, E. poeppigiana y Musa spp. (Musaceae), en donde se manejan 314 y 407 individuos por $\mathrm{ha}^{-1}$ de cada especie, respectivamente (Tully, Wood, \& Lawrence, 2013). Particularmente, Musa spp. presenta una altura que varía de 5 a $7 \mathrm{~m}$ (Somarriba et al., 2004). CPL al combinar E. poeppigiana y la especie nativa C. alliodora (Boraginaceae), mantiene una media de 264 y 136 árboles ha ${ }^{-1}$ de cada especie, respectivamente, en donde $C$. alliodora presenta una altura media de $10.6 \mathrm{~m}$ (Florian, 2005).

Muestreo de mariposas: $\mathrm{Al}$ centro de los cafetales y FR se establecieron tres transectos paralelos con una longitud de $80 \mathrm{~m} \times 4 \mathrm{~m}$ cada uno, y distanciados a $25 \mathrm{~m}$ entre los mismos (adaptado de Caldas \& Robbins, 2003). El tamaño de los transectos se determinó por la superficie mínima de 1 ha de los cafetales. Se realizó un premuestreo de mariposas en abril de 2007 mediante captura y liberación usando una red entomológica y guías de campo ilustradas (DeVries 1987, 1997; Chacón \& Montero, 2007). Esto se realizó para contar con un inventario y entrenamiento preliminar. Cada transecto se recorrió durante $30 \mathrm{~min}$ (1.5 horas/sitio) entre las 8:00 y 15:00 horas en días soleados. Todos las sitios se muestrearon una vez por mes desde mayo a julio de 2007. El esfuerzo de muestreo fue de 4.5 horas por sitio; 27 horas en cada SAF y FR. Se registraron los individuos adultos de mariposas mediante observación directa dentro de los límites de los transectos. Los individuos de difícil identificación durante el vuelo o desconocidos, fueron capturados con una red entomológica y posteriormente liberados. Posteriormente, las especies registradas se clasificaron por gremios alimenticios (nectarívoros y frugívoros) y por preferencias de hábitat (especies de áreas abiertas y de áreas cerradas) de acuerdo con DeVries $(1987,1997)$ y Chacón y Montero (2007).
Para diferenciar la composición de especies de mariposas entre los cuatro sistemas evaluados, se realizó un análisis de conglomerados jerárquico mediante el índice de disimilitud de Jaccard modificado por Chao, Chazdon, Colwell y Shen (2005); con el método de ligamiento completo (Jain, Murty, \& Flynn, 1999) usando el paquete 'vegan' de R (R Development Core Team, 2013; Oksanen et al., 2016). El valor del índice varía de 0 (máxima similitud) a 1 (máxima disimilitud). Adicionalmente, se realizó un análisis de especies indicadoras mediante el método de Dufrêne y Legendre (1997), a través del paquete 'indicspecies' de R (De Cáceres \& Legendre, 2009; R Development Core Team, 2013). Las especies indicadoras determinan la exclusividad (A) y fidelidad (B) de cierta(s) especie(s) a cierto grupo o hábitat mediante un índice de valor indicador (IndVal $=\mathrm{A} \times \mathrm{B})$. El valor A es la abundancia media de una especie en un grupo, B es la frecuencia relativa de ocurrencia de la especie en un grupo o hábitat en particular. IndVal puede variar de 0 a 1 , en donde 1 es el indicador perfecto de una especie en un grupo. Este análisis selecciona el IndVal máximo de las especies para evaluar su significancia estadística mediante una prueba de Monte Carlo (1000 aleatorizaciones). Por otra parte, se hizo una comparación de especies entre los hábitats mediante curvas de acumulación de especies interpoladas basadas en el número de individuos (rarefacción con 1000 aleatorizaciones) con el programa EstimateS 9.1 (Colwell, 2013). Así mismo, se determinó el nivel de inventario en cada hábitat, basado en el porcentaje de especies observadas en relación con las especies estimadas bajo el modelo de Clench, usando 100 iteraciones (Soberón \& Llorente, 1993) mediante el programa InfoStat (Di Rienzo et al., 2013). Entre los SAF y FR, se comparó la abundancia, riqueza y diversidad verdadera con el exponencial del índice de Shannon $\left(\operatorname{Exp}\left(H^{\prime}\right)\right)($ Jost, 2006) de mariposas nectarívoras, frugívoras, de áreas abiertas y de áreas cerradas. Previa comprobación de la normalidad de los datos con la prueba de Shapiro-Wilks; este análisis se realizó a través de un análisis de varianza (ANDEVA) de una 
vía y comparación de medias de LSD de Fisher (Carmer \& Swanson, 1973), para un diseño completamente aleatorizado con el programa InfoStat (Di Rienzo et al., 2013).

\section{RESULTADOS}

Se registraron 1259 individuos de mariposas de 106 especies, 15 subfamilias y cinco familias. Del total de individuos registrados, 280 fueron en CP, 350 en CPM, 303 en CPL y 326 en FR pertenecientes a 35, 45, 38 y 65 especies, respectivamente. Las subfamilias Satyrinae y Coliadinae fueron las más comunes por su número de individuos y especies en los SAF con café. Las subfamilias Ithomiinae, Satyrinae y Heliconiinae fueron las más representativas por su abundancia y número de especies en los FR.

Composición de las especies de mariposas: Mediante el análisis de conglomerados jerárquico, las especies de mariposas registradas en las 24 parcelas estuvieron asociadas con tres grupos diferentes. Las especies presentes en todas las parcelas de FR fueron exclusivas del grupo uno (G1). Las especies de todas las parcelas de CPM y la mayoría de CP se concentraron al grupo dos (G2). Finalmente, casi la totalidad de las parcelas de CPL estuvieron aglomeradas al grupo tres (G3) (Fig. 1).

Mediante el análisis de especies indicadoras, se determinaron tres grupos que coincidieron con los tres grupos determinados en el análisis de conglomerados. Un grupo de especies (G1) estuvo conformado por especies típicas de bosques. Un segundo grupo distintivo (G2) estuvo caracterizado por especies afines a áreas abiertas o perturbadas. Finalmente, un tercer grupo (G3) estuvo representado por especies propias de bosques secundarios jóvenes y bosques perturbados, sobre todo las especies de mayor valor indicador (Cuadro 1).

De acuerdo con las curvas de rarefacción, la riqueza de mariposas estandarizada a un mismo número de individuos fue similar entre los SAF CP, CPM y CPL (Fig. 2). Por la clara separación de las curvas de acumulación entre
FR y los SAF, la riqueza de mariposas fue mayor en los FR que en los SAF (Fig. 2). Con el estimador de riqueza de especies basado en el modelo de Clench, se determinó que se esperan 49 especies de mariposas en CP, 65 especies en CPM, 51 especies en CPL y 99 especies en FR. Con las especies observadas y las especies estimadas con el modelo de Clench, se determinó un nivel de inventario del $71 \%$ en $\mathrm{CP}$, un $69 \%$ en CPM, un $75 \%$ en CPL y un $66 \%$ en FR.

Los gremios alimenticios y preferencias de hábitat de las mariposas: Las mariposas nectarívoras y las de áreas abiertas, tuvieron una abundancia, riqueza y diversidad semejantes entre los tres SAF con café (Cuadro 2). Así

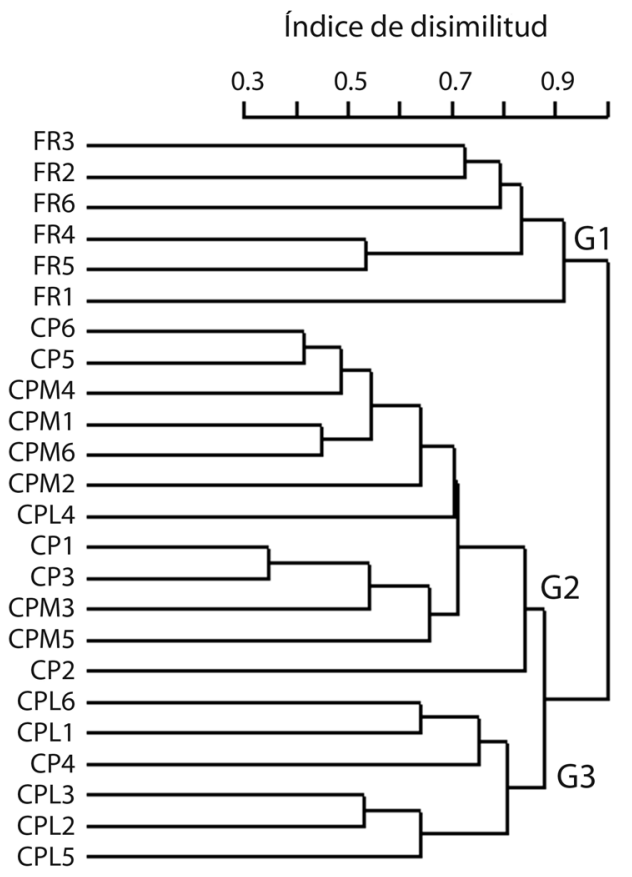

Fig. 1. Dendrograma basado en el índice de disimilitud de Jaccard modificado por Chao et al. (2005) y método de enlace completo de la comunidad de mariposas presentes en los sistemas café-poró (CP), café-poró-musa (CPM), café-poró-laurel (CPL) y fragmentos de bosque (FR).

Fig. 1. Cluster dendrogram (Jaccard dissimilarity index modified by Chao et al. (2005) and the complete linkage method) of butterfly species similarity in the coffee-poró (CP), coffee-poró-musa (CPM), and coffee-poró-laurel (CPL) systems, and forest fragments (FR). 


\section{CUADRO 1}

Especies indicadoras significativas $(\mathrm{P}<0.05)$ y sus valores indicadores (IndVal) de mariposas asociadas con los sistemas café-poró (CP), café-poró-musa (CPM), café-poró-laurel (CPL) y fragmentos de bosque (FR).

$$
n=6 \text { parcelas por sistema agroforestal }
$$

TABLE 1

Significative indicator species of butterflies $(\mathrm{P}<0.05)$ and their indicator value (IndVal) associated with the coffee-poró (CP), coffee-poró-musa (CPM), and coffee-poró-laurel (CPL) systems, and forest fragments (FR). $n=6$ plots per agroforestry system

\begin{tabular}{|c|c|c|c|c|}
\hline Grupo & Hábitat & Especie & Subfamilia & IndVal \\
\hline \multirow[t]{13}{*}{ G1 } & FR & Heliconius cydno galanthus Bates, 1864 & Heliconiinae & $0.97 * *$ \\
\hline & & Pseudoscada timna pusio Godman \& Salvin, 1869 & Ithomiinae & $1.00 * *$ \\
\hline & & Hypoleria lavinia cassotis Bates, 1864 & Ithomiinae & $0.91 * *$ \\
\hline & & Napeogenes tolosa amara Godman, 1899 & Ithomiinae & $0.82 * *$ \\
\hline & & Oleria paula Weymer, 1884 & Ithomiinae & $0.82 * *$ \\
\hline & & Dircenna dero euchytma Felder \& Felder, 1867 & Ithomiinae & $0.71 *$ \\
\hline & & Hyposcada virginiana evanides Haensch, 1909 & Ithomiinae & $0.71 *$ \\
\hline & & Melinaea ethra lilis Bates, 1864 & Ithomiinae & $0.71 *$ \\
\hline & & Pteronymia notilla Butler \& Druce, 1872 & Ithomiinae & $0.71 *$ \\
\hline & & Caligo eurilochus Cramer, 1775 & Morphinae & $0.91 * *$ \\
\hline & & Morpho peleides limpida Butler, 1872 & Morphinae & $0.91 * *$ \\
\hline & & Pierella helvina incanescens Godman \& Salvin, 1877 & Satyrinae & $0.97 * *$ \\
\hline & & Cithaerias menander Drury, 1782 & Satyrinae & $0.82 * *$ \\
\hline \multirow[t]{3}{*}{$\mathrm{G} 2$} & CP y CPM & Anartia jatrophae Linnaeus, 1763 & Nymphalinae & $0.71 *$ \\
\hline & & Cissia pompilia Felder \& Felder, 1867 & Satyrinae & $0.76^{* *}$ \\
\hline & & Hermeuptychia hermes Fabricius, 1775 & Satyrinae & $0.68 *$ \\
\hline \multirow[t]{3}{*}{ G3 } & $\mathrm{CPL}$ & Heliconius erato petiverana Doubleday, 1847 & Heliconiinae & $0.74 *$ \\
\hline & & Ascia monuste Linnaeus, 1764 & Pierinae & $0.66^{*}$ \\
\hline & & Pareuptychia ocirrhoe Fabricius, 1789 & Satyrinae & $0.72 *$ \\
\hline
\end{tabular}

$*=\mathrm{P}<0.05, * *=\mathrm{P}<0.01$.

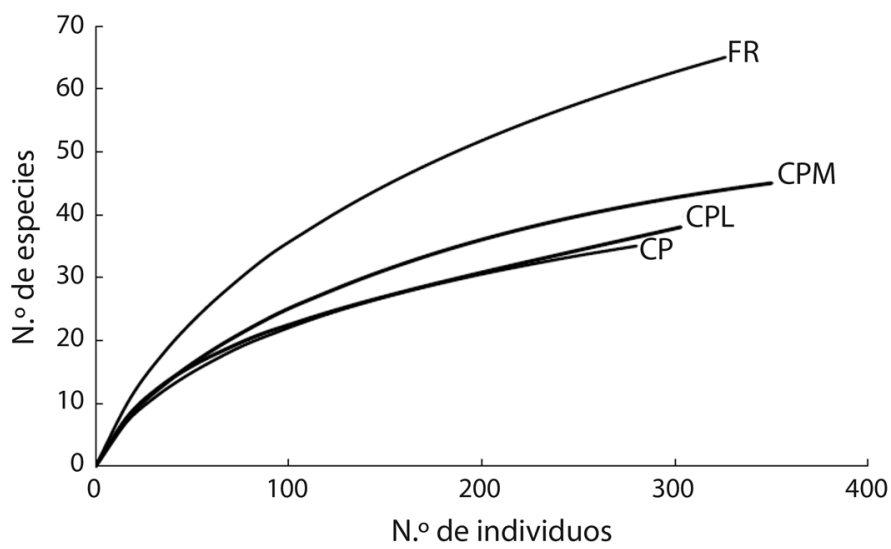

Fig. 2. Curvas de rarefacción de especies estimadas de mariposas basadas en el número de individuos registrados en los sistemas café-poró (CP), café-poró-musa (CPM), café-poró-laurel (CPL) y fragmentos de bosque (FR).

Fig. 2. Individual-based rarefaction curves for butterfly species recorded in the coffee-poró (CP), coffee-poró-musa (CPM), and coffee-poró-laurel (CPL) systems, and forest fragments (FR). 
CUADRO 2

La abundancia, riqueza y diversidad media ( \pm desviación estándar) de mariposas por gremios alimenticios y preferencias de hábitat en los sistemas café-poró (CP), café-poró-musa (CPM), café-poró-laurel (CPL) y fragmentos de bosque (FR). $n=6$ parcelas por sistema agroforestal.

TABLE 2

The mean value ( \pm standard deviation) of abundance, species richness, and diversity of butterflies by feeding guilds and habitat preferences in the coffee-poró (CP), coffee-poró-musa (CPM), and coffee-poró-laurel (CPL) systems, and forest fragments (FR). $n=6$ plots per agroforestry system.

\begin{tabular}{|c|c|c|c|c|c|}
\hline Variable & $\mathrm{CP}$ & CPM & CPL & FR & Valor P \\
\hline \multicolumn{6}{|l|}{ Especies nectarívoras } \\
\hline Abundancia & $26.3 \pm 13.2 \mathrm{a}$ & $25.8 \pm 9.2 \mathrm{a}$ & $36.2 \pm 20.5 \mathrm{a}$ & $40.8 \pm 15.1 \mathrm{a}$ & 0.2550 \\
\hline Riqueza & $10.2 \pm 2.6 \mathrm{~b}$ & $12.5 \pm 2.3 \mathrm{ab}$ & $11.0 \pm 3.2 \mathrm{~b}$ & $16.2 \pm 4.4 \mathrm{a}$ & 0.0206 \\
\hline Diversidad $\operatorname{Exp}\left(H^{\prime}\right)$ & $7.2 \pm 2.2 b$ & $9.9 \pm 1.4 \mathrm{ab}$ & $7.6 \pm 1.8 \mathrm{~b}$ & $12.5 \pm 3.7 \mathrm{a}$ & 0.0037 \\
\hline \multicolumn{6}{|l|}{ Especies frugívoras } \\
\hline Abundancia & $20.3 \pm 8.2 b$ & $32.5 \pm 13.6 \mathrm{a}$ & $14.3 \pm 7.4 \mathrm{~b}$ & $13.5 \pm 4.8 b$ & 0.0060 \\
\hline Riqueza & $3.3 \pm 0.8 \mathrm{a}$ & $4.8 \pm 1.7 \mathrm{a}$ & $4.3 \pm 1.6 \mathrm{a}$ & $5.2 \pm 1.2 \mathrm{a}$ & 0.1464 \\
\hline Diversidad $\operatorname{Exp}\left(H^{\prime}\right)$ & $2.3 \pm 0.4 \mathrm{c}$ & $2.9 \pm 0.8 \mathrm{bc}$ & $3.3 \pm 0.8 \mathrm{ab}$ & $3.9 \pm 0.4 \mathrm{a}$ & 0.0023 \\
\hline \multicolumn{6}{|c|}{ Especies de áreas abiertas } \\
\hline Abundancia & $15.0 \pm 3.0 \mathrm{a}$ & $18.0 \pm 9.3 \mathrm{a}$ & $24.0 \pm 14.0 \mathrm{a}$ & $0.8 \pm 0.8 \mathrm{~b}$ & 0.0011 \\
\hline Riqueza & $6.7 \pm 1.0 \mathrm{a}$ & $7.5 \pm 1.9 \mathrm{a}$ & $6.7 \pm 1.9 \mathrm{a}$ & $0.7 \pm 0.5 \mathrm{~b}$ & $<0.0001$ \\
\hline Diversidad $\operatorname{Exp}\left(H^{\prime}\right)$ & $5.0 \pm 1.1 \mathrm{a}$ & $5.9 \pm 1.2 \mathrm{a}$ & $4.9 \pm 1.4 \mathrm{a}$ & $1.0 \pm 0.0 \mathrm{~b}$ & $<0.0001$ \\
\hline \multicolumn{6}{|c|}{ Especies de áreas cerradas } \\
\hline Abundancia & $15.8 \pm 10.6 \mathrm{~b}$ & $18.5 \pm 7.7 \mathrm{~b}$ & $12.5 \pm 3.7 \mathrm{~b}$ & $49.0 \pm 13.3 \mathrm{a}$ & $<0.0001$ \\
\hline Riqueza & $4.3 \pm 1.9 b$ & $7.0 \pm 1.8 \mathrm{~b}$ & $6.5 \pm 1.9 \mathrm{~b}$ & $19.3 \pm 3.3 \mathrm{a}$ & $<0.0001$ \\
\hline Diversidad $\operatorname{Exp}\left(H^{\prime}\right)$ & $2.9 \pm 0.1 \mathrm{c}$ & $4.5 \pm 1.0 \mathrm{bc}$ & $5.7 \pm 1.5 \mathrm{~b}$ & $15.0 \pm 2.8 \mathrm{a}$ & $<0.0001$ \\
\hline
\end{tabular}

Letras distintas entre los hábitats indican diferencias significativas con base en la prueba de LSD de Fisher $(\mathrm{P}<0.05)$.

mismo, la riqueza de especies frugívoras y la de especies de áreas cerradas fueron similares entre los SAF. En contraste, CPM resultó con la mayor abundancia de especies frugívoras. Así mismo, CPL resultó con la mayor diversidad de especies frugívoras y diversidad de especies de áreas cerradas.

En la comparación de los SAF con los FR, hubo diferencias significativas en la riqueza y diversidad de especies nectarívoras, la diversidad de especies frugívoras y la abundancia, riqueza y diversidad de especies de áreas abiertas y cerradas (Cuadro 2). En particular, FR resultó con la mayor riqueza y diversidad de especies nectarívoras, diversidad de especies frugívoras y abundancia, riqueza y diversidad de especies de áreas cerradas que los SAF con café. Por el contrario, FR resultó con la menor abundancia, riqueza y diversidad de especies de áreas abiertas en comparación con los SAF.

\section{DISCUSIÓN}

Los resultados sugieren que entre los SAF con café de sombra arbórea exótica monoespecífica (CP) y en combinación con Musa spp. (CPM), o una especie arbórea nativa (Cordia alliodora) (CPL), pueden influir de manera distinta en la diversidad de mariposas diurnas de paisajes fragmentados. Aunque los FR fueron de mayor importancia para la conservación de especies típicas de bosques continuos (DeVries 1987, 1997), CPL y CPM en comparación con $\mathrm{CP}$, significaron de mayor importancia para la conservación de mariposas en bosques fragmentados. Los resultados son consistentes con estudios comparativos entre SAF modernos con café y bosques (Horner-Devine, Daily, \& Ehrlich, 2003; Francesconi, Nair, Levey, Daniels, \& Cullen, 2013). Sin embargo, nuestro estudio sugiere que la combinación de dos 
especies de sombra, en este caso ya sea CPM o CPL; es mejor para la conservación de mariposas diurnas que los cafetales con sombra monoespecífica $(\mathrm{CP})$.

Las diferencias detectadas en la composición de especies de mariposas de los SAF con café, pueden estar relacionadas con las diferencias en la estructura y composición de la sombra de los cafetales (Somarriba et al., 2004). En este sentido, la distinta composición de especies de mariposas entre CPL y los demás SAF; y la mayor diversidad $\left(\operatorname{Exp}\left(H^{\prime}\right)\right)$ de especies de áreas cerradas en CPL que en CP, pueden asociarse con las diferencias en los estratos de sombra. La presencia en CPL de la especie nativa $C$. alliodora en el estrato alto de la sombra y la especie exótica E. poeppigiana en el estrato bajo, pueden crear condiciones microambientales favorables para especies de mariposas de áreas cerradas (Muriel \& Kattan, 2009). De hecho, las especies indicadoras de CPL, $H$. erato petiverana y $P$. ocirrhoe, requieren de hábitats con cierta complejidad estructural y florística, como los bosques perturbados, bosques ribereños y bordes de bosques (DeVries, 1987; Chacón \& Montero, 2007; Finkbeiner, 2014). A diferencia de CP y CPM, que se caracterizan por presentar un estrato bajo de sombra dominado por la especie arbórea exótica E. poeppigiana. Además, E. poeppigiana es manejada con frecuentes podas y descopes para aportar materia orgánica al suelo. Esto puede crear condiciones de alta radiación solar en los cafetales (Somarriba et al., 2004); lo que podría explicar la baja diversidad $\left(\operatorname{Exp}\left(H^{\prime}\right)\right)$ de mariposas de áreas cerradas y la presencia de especies indicadoras de áreas abiertas en $\mathrm{CP}$ y CPM. Por otra parte, la alta abundancia de mariposas frugívoras en CPM puede estar relacionada con la presencia de Musa spp. en este SAF en particular. Los frutos de Musa spp., disponibles durante todo el año en la zona (C. Cerdán, comunicación personal, junio 2017), son una fuente alimenticia importante para mariposas frugívoras (DeVries, 1987). Además, Musa spp. son huéspedes importantes para mariposas frugívoras de la subfamilia Morphinae, propia de bosques continuos (DeVries, 1987; Chacón
\& Montero, 2007). Debido al escaso manejo agronómico que recibe Musa spp. en la zona (observación personal), CPM puede ofrecer recursos alimenticios y plantas huéspedes para mariposas frugívoras asociadas con los fragmentos de bosque cercanos tal y como sugiere Munyuli (2013).

Finalmente, a diferencia de CP, CPL y CPM pueden crear una matriz más favorable para la conservación de mariposas de los FR, dado que CPL albergó especies indicadoras asociadas con los FR, además, CPL y CPM albergaron la mayor diversidad de especies de áreas cerradas y abundancia de frugívoras, respectivamente. Por el contrario, $\mathrm{CP}$, el SAF más simple en su estructura y composición de sombra, puede representar una barrera para las especies de bosque que evitan las áreas abiertas (Muriel \& Kattan, 2009). Además, $\mathrm{CP}$ puede representar un hábitat pobre para las especies de mariposas con requerimientos alimenticios particulares como las frugívoras (Mas \& Dietsch, 2003; Perfecto, Mas, Dietsch, \& Vandermeer, 2003).

\section{AGRADECIMIENTOS}

Al programa de becas de la Fundación Ford por su apoyo para la realización de esta investigación. A Fernando Casanoves y Alexis Pérez de la unidad de biometría y de agroforestería del CATIE, respectivamente, por su apoyo en el diseño experimental y acompañamiento en campo. A Carlos Cerdán por la información brindada acerca de la dinámica de producción de Musa spp. en el CBVCT. A los caficultores $\mathrm{y}$ propietarios de fragmentos de bosques del CBVCT quienes amablemente nos permitieron entrar a sus fincas.

\section{RESUMEN}

El cambio de uso del suelo por la intensificación de la agricultura contribuye a la fragmentación de los bosques tropicales y la pérdida de la biodiversidad. En este sentido, a diferencia de los sistemas agroforestales (SAF) tradicionales, los SAF modernos son considerados de poca importancia para la conservación de la biodiversidad de los fragmentos de bosque. Sin embargo, existe una 
tendencia al establecimiento de SAF modernos. Debido a la sensibilidad a los cambios ambientales, se evaluó la diversidad de mariposas en SAF modernos de Coffea arabica y Erythrina poeppigiana (CP) y en combinación con Musa spp. (CPM) o Cordia alliodora (la única especie nativa de los SAF) (CPL), además de fragmentos de bosque muy húmedo premontano (FR) en el Corredor Biológico Volcánica Central-Talamanca, Costa Rica. En seis sitios representativos de cada sistema se establecieron tres transectos paralelos de $80 \mathrm{~m}$ cada uno y distanciados a $25 \mathrm{~m}$ entre los mismos. Cada cafetal y fragmento de bosque fue muestreado durante 1.5 horas en total para el registro de mariposas. Durante tres fechas de muestreo, se obtuvo un esfuerzo de muestreo de 4.5 horas/sitio y 27 horas en cada SAF y en FR. CP y CPM juntos tuvieron una composición de especies de mariposas diferente a la de CPL y FR. CP y CPM juntos resultaron con las especies de áreas abiertas Hermeuptychia hermes y Anartia jatrophae como indicadoras. CPL resultó con las especies de bosques secundarios Heliconus erato petiverana y Pareuptychia ocirrhoe como indicadoras. Por el contrario, FR resultó con diversas especies indicadoras típicas de bosques continuos tales como Pierella helvina incanescens, Caligo eurilochus y Morpho peleides limpida. Además, la riqueza de especies en FR fue mayor en relación a los SAF. Sin embargo, entre los SAF, CPL resultó con la mayor diversidad $\left(\operatorname{Exp}\left(H^{\prime}\right)\right)$ de especies de áreas cerradas que CP. Así mismo, CPM tuvo una mayor abundancia de mariposas frugívoras que CP y CPL. En bosques fragmentados, los cafetales con sombras exóticas pueden ser mejores hábitats para mariposas de bosques si se combinan con árboles nativos $\mathrm{y} / \mathrm{o}$ frutales.

Palabras clave: especies indicadoras; gremios; matriz de cafetales; Papilionoidea; preferencias de hábitat; sistemas agroforestales.

\section{REFERENCIAS}

Bonebrake, T. C., Ponisio, L. C., Boggs, C. L., \& Ehrlich, P. R. (2010). More than just indicators: A review of tropical butterfly ecology and conservation. Biological Conservation, 143(8), 1831-1841.

Caldas, A., \& Robbins, R. K. (2003). Modified pollard transects for assessing tropical butterfly abundance and diversity. Biological Conservation, 110(2), 211-219.

Canet, L. (2003). Ficha técnica del corredor biológico Turrialba-Jiménez. Costa Rica: Escuela de Ciencias Ambientales.

Carmer, S. G., \& Swanson, M. R. (1973). An evaluation of ten pairwise multiple comparison procedures by Monte Carlo methods. Journal of the American Statistical Association, 68(341), 66-74.

Chacón, I., \& Montero, J. (2007). Mariposas de Costa Rica. Santo Domingo de Heredia, Costa Rica: INBIO.
Chao, A., Chazdon, R. L., Colwell, R. K., \& Shen, T. (2005). A new statistical approach for assessing similarity of species composition with incidence and abundance data. Ecology Letters, 8, 148-159.

Colwell, R. K. (2013). EstimateS Version 9.1: Statistical estimation of species richness and shared species from samples. Recuperado de http:viceroy.eeb.uconn. edu/estimates

De Beenhouwer, M., Aerts, R., \& Honnay, O. (2013). A global meta-analysis of the biodiversity and ecosystem service benefits of coffee and cacao agroforestry. Agriculture, Ecosystems and Environment, 175, 1-7.

De Cáceres, M., \& Legendre, P. (2009). Associations between species and groups of sites: Indices and statistical inference. R Package Version 1.7.1. Recuperado de http://cran.r-project.org/web/packages/indicspecies/index.html

DeVries, P. J. (1987). The butterflies of Costa Rica and their natural history. Volume I: Papilionidae, Pieridae, Nymphalidae. New Jersey: Princeton University Press.

DeVries, P. J. (1997). The butterflies of Costa Rica and their natural history. Volume II: Riodinidae. New Jersey: Princeton University Press.

Di Rienzo, J. A., Casanoves, F., Balzarini, M. G., Gonzalez, L., Tablada, M., \& Robledo, C. W. (2013). InfoStat Version 2013. Grupo InfoStat-Universidad Nacional de Córdoba, Argentina. Recuperado de http://www. infostat.com.ar

Dobrovolski, R., Diniz-Filho, J. A. F., Loyola, R. D., \& De Marco Júnior, P. (2011). Agricultural expansion and the fate of global conservation priorities. Biodiversity and Conservation, 20 (11), 2445-2459.

Dufrêne, M., \& Legendre, P. (1997). Species assemblages and indicator species: The need for a flexible asymmetrical approach. Ecological Monographs, 67, 345-366.

Finkbeiner, S. D. (2014). Communal roosting in Heliconius butterflies (Nymphalidae): Roost recruitment, establishment, fidelity, and resource use trends based on age and sex. Journal of the Lepidopterists'Society, 68(1), 10-16.

Florian, E. M. (2005). Tropical bird assemblages in coffee agroforestry systems: Exploring the relationships between landscape context, structural complexity and bird communities in the Turrialba-Jiménez Biological Corridor, Costa Rica. (Tesis de Maestría). Recuperado de http://repositorio.bibliotecaorton.catie.ac.cr/ handle/11554/1

Francesconi, W., Nair, P. K. R., Levey, D. J., Daniels, J., \& Cullen, L. (2013). Butterfly distribution in fragmented landscapes containing agroforestry practices 
in Southeastern Brazil. Agroforestry Systems, 87(6), $1321-1338$

Horner-Devine, M. C., Daily, G. C., \& Ehrlich P. R. (2003) Countryside biogeography of tropical butterflies. Conservation Biology, 17(1), 168-177.

Jain, A., Murty, M., \& Flynn, P. (1999). Data clustering: A review. ACM Computing Surveys (CSUR), 31(3), 264-323.

Jha, S., Bacon, C., Philpott, S., Mendez, V., Laderach, P., \& Rice, R. (2014). Shade coffee: Update on a disappearing refuge for biodiversity. Bioscience 64(5), 416-428.

Jost, L. (2006). Entropy and diversity. Oikos, 113, 363-375

Kremen, C. (2015). Reframing the land-sparing/landsharing debate for biodiversity conservation. Annals of the New York Academy of Sciences, 1355, 52-76.

Mas, A. H., \& Dietsch, T. V. (2003). An index of management intensity for coffee agroecosystems to evaluate butterfly species richness. Ecological Applications, 13(5), 1491-1501.

Munyuli, M. B. T. (2013). Drivers of species richness and abundance of butterflies in coffee-banana agroforests in Uganda. International Journal of Biodiversity Science, Ecosystem Services \& Management, 9(4), 298-310.

Muriel, S. B., \& Kattan, G. H. (2009). Effects of patch size and type of coffee matrix on Ithomiine butterfly diversity and dispersal in cloud-forest fragments. Conservation Biology, 23(4), 948-956.

Murrieta, E. (2006). Caracterización de cobertura vegetal y propuesta de una red de conectividad ecológica en el Corredor Biológico Volcánica Central-Talamanca,
Costa Rica. (Tesis de Maestría). Recuperado de http://orton.catie.ac.cr/repdoc/A0739e/A0739e.pdf

Myers, N., Mittermeier, R. A., Mittermeier, C. G., \& da Fonseca, G. A. B. (2000). Biodiversity hotspots for conservation priorities. Nature, 403, 853-858.

Oksanen, J., Guillaume-Blanchet, F., Kindt, R., Legendre, P., Peter-R, M., O'Hara, R. B., Simpson, G. L., Solymos, P., Stevens, M. H. H., \& Wagner, H. (2016). Vegan: Community Ecology Package. R package version 2.3-5. Recuperado de http://CRAN.R-project. org/package= $=$ vegan

Perfecto, I., Mas, A., Dietsch, T., \& Vandermeer, J. (2003). Conservation of biodiversity in coffee agroecosystems: A tri-taxa comparison in southern Mexico. Biodiversity and Conservation, 12(6), 1239-1252.

R Development Core Team. (2013). R: A language and environment for statistical computing. $\mathrm{R}$ foundation for statistical computing, Vienna, Austria. Recuperado de http://www.R-project.org

Soberón, J. M., \& Llorente J. B. (1993). The use of species accumulation functions for the prediction of species richness. Conservation Biology, 7, 480-488.

Somarriba, E., Harvey, C. A., Samper, M., Anthony, F., González, J., Staver, C., \& Rice, R. A. (2004). Biodiversity conservation in neotropical coffee (Coffea arabica) plantations. In G. Schroth, G. A. B. da Fonseca, C. A. Harvey, C. Gascon, H. L. Vasconcelos, \& A. M. N. Izac (Eds.), Agroforestry and biodiversity conservation in tropical landscapes (pp. 198-226). Washington, DC: Island Press.

Tully, K. L., Wood, S. A., \& Lawrence, D. (2013). Fertilizer type and species composition affect leachate nutrient concentrations in coffee agroecosystems. Agroforestry Systems 87(5), 1083-1100. 